\title{
Sites of Microtubule Stabilization for the Axon
}

\author{
Peter W. Baas, ${ }^{1}$ Fridoon J. Ahmad, ${ }^{1}$ Thomas P. Pienkowski, ${ }^{1}$ Anthony Brown, ${ }^{2}$ and Mark M. Black ${ }^{2}$ \\ ${ }^{1}$ Department of Anatomy, The University of Wisconsin Medical School, Madison, Wisconsin 53706 and ${ }^{2}$ Department of \\ Anatomy and Cell Biology, Temple University School of Medicine, Philadelphia, Pennsylvania 19140
}

\begin{abstract}
We have sought to determine the principal site(s) in the neuron where axonal microtubules (MTs) are stabilized. To accomplish this, we compared the proximal and distal regions of the axon and the axon shaft with regard to their content of newly stabilized MT polymer, using the following criteria. Stable polymer was identified by its resistance to nocodazole, and newly stabilized polymer was distinguished from older stable polymer by the staining of the former but not the latter for tyrosinated $\alpha$-tubulin. Our results indicate that roughly $36.4 \%, 5.4 \%$, and $2.4 \%$ of the total MT mass in the proximal and distal regions of the axon and the axon shaft is newly stabilized, respectively. Thus, while MT stabilization occurs throughout the axon, the proximal region is by far the most active with regard to this process.
\end{abstract}

[Key words: neuron, axon growth, microtubule stability, tyrosinated $\alpha$-tubulin]

The microtubule (MT) array of the axon consists of two types of polymer, stable and labile, that differ in their rates of druginduced depolymerization, solubility properties, and presumably, rates of subunit turnover (Black et al., 1984; Brady et al., 1984; Morris and Lasek, 1984; Ferreira et al., 1989; Baas and Black, 1990; Baas et al., 1991; Baas and Ahmad, 1992). The proportion of stable polymer is particularly high in axons compared to non-neuronal cells, and this presumably relates to the unique morphologic constraints inherent in the elongate geometry of the axon. In addition, it is now well established that the stable MT polymer has an essential role in regulating MT dynamics in the axon. The plus ends of stable MTs represent the exclusive sites for MT assembly in the axon, and hence serve as nucleating structures for MT assembly in the axon (Baas and Ahmad, 1992; see also Baas and Black, 1990). MTs are intrinsically labile structures, and stability is conferred upon them subsequent to their assembly (Kirschner and Mitchison, 1986). Thus, in growing axons, newly assembled MT polymer is initially labile, and portions of this are converted to stable polymer, thereby increasing the stable MT mass of the axon. Consistent with this view, several lines of evidence indicate that the levels and proportion of stable polymer increase during neuronal de-

\footnotetext{
Recived Scpt. 24, 1992; revised Nov. 13, 1992; accepted Nov. 23, 1992.

We thank Ms. Janet Krotzman and the Blood Bank at St. Marys Hospital in Madison, WI for generously supplying human placental serum for our cultures, and The National Cancer Institute for their generous gift of taxol. This work was supported by NIH Grants NS 28785 to P.W.B. and NS 23530 to M.M.B. and NSF Grant IBN-9209939 to P.W.B. P.W.B. is the recipient of a Research Career Development Award from NIH.

Correspondence should be addressed to Peter W. Baas, Department of Anatomy, The University of Wisconsin Medical School, 1300 University Avenue, Madison, WI 53706.

Copyright (C) 1993 Society for Neuroscience $0270-6474 / 93 / 132177-09 \$ 05.00 / 0$
}

velopment (Black and Greene, 1982; Ferreira et al., 1989; Lim et al., 1989; Arregui et al., 1991). Despite the functional significance of the conversion from labile to stable polymer, a process termed MT stabilization, surprisingly little is known about how or where it occurs.

In the present study, we have sought to determine the principal site(s) in the neuron where axonal MTs are stabilized. Different possibilities for where axonal MTs are stabilized have decidedly different implications for the mechanisms by which the entire MT array of the axon is elaborated during axon growth. Some models for axon growth stress that MT assembly and stabilization occur principally behind the advancing growth cone (Bamburg et al., 1986; Mitchison and Kirschner, 1988), while other models stress that MTs are assembled and stabilized principally proximally, and are then translocated distally by an active transport mechanism (Lasek, 1982, 1986, 1988; Reinsch et al., 1991). Determination of sites of MT stabilization will assist in evaluating the merits of these very different models.

In our previous studies on the stability properties of the MTs in cultured sympathetic neurons, we determined that axons contain both drug-stable and drug-labile MT polymer that differ in their rates of drug-induced depolymerization by roughly 35fold (Baas and Black, 1990; Baas et al., 1991). Drug-stable polymer represents a substantial portion of the MT mass of these axons, accounting for $\approx 55 \%$ of the polymer mass in the axon shaft. To explore where drug stability is conferred on axonal MTs, we have developed a strategy for quantifying the levels of newly stabilized MT polymer in different regions of the axon. Although indirect, this strategy is based on current understanding of the composition and stability properties of MTs in ncuronal and non-neuronal cells. In axons of sympathetic neurons, the drug-labile polymer can be visually distinguished from the vast majority of the drug-stable polymer by the preferential staining of the former for tyrosinated $\alpha$-tubulin (Baas and Black, 1990; Baas et al., 1991; Baas and Ahmad, 1992; Ahmad et al., 1993). The relative levels of tyrosinated $\alpha$-tubulins in polymer are regulated posttranslationally. Most $\alpha$-tubulins are synthesized with a tyrosine residue at their C-terminus, and this residue can be removed posttranslationally by tubulin carboxypeptidase and then replaced by tubulin tyrosine ligase (for reviews, see Barra et al., 1988; Bulinski and Gunderson, 1991). The tubulin carboxypeptidase acts on polymerized $\alpha$-tubulin, whereas the tubulin ligase acts on unassembled $\alpha$-tubulin, rapidly retyrosinating any $\alpha$-tubulin released from MIs. Consequently, detyrosinated $\alpha$-tubulin is found almost exclusively in polymer, and the relative content of detyrosinated $\alpha$-tubulin in MTs increases progressively with their age (Gunderson et al., 1987; Khawaja et al., 1988; Webster et al., 1990). Because the amount of detyrosinated $\alpha$-tubulin in an MT increases at the expense of its 
tyrosinated $\alpha$-tubulin subunits, the relative content of tyrosinated $\alpha$-tubulin in polymer decreases progressively with polymer age. Thus, we can infer that, in axons, the drug-stable polymer, which stains poorly or not at all for tyrosinated $\alpha$-tubulin, is older than the drug-labile polymer, which stains strongly for tyrosinated $\alpha$-tubulin. Because drug-stable polymer is thought to be generated from drug-labile polymer, we would expect that the most recently stabilized polymer should still contain relatively high levels of tyrosinated $\alpha$-tubulin. Therefore, we reasoned that newly stabilized polymer should correspond to the MT polymer in the axon that is both stable and tyrosinated.

In the present study, we have sought to identify the region(s) of the axon that contains the highest levels of newly stabilized polymer. Specifically, we quantified the levels of drug-labile, drug-stable, and newly stabilized polymer in three key regions of the axon: the $10 \mu \mathrm{m}$ closest to the cell body (proximal axon), the $10 \mu \mathrm{m}$ closest to the growth cone (distal axon), and the axon shaft, more than $100 \mu \mathrm{m}$ from both the cell body and the growth cone. Drug-stable polymer was distinguished from drug-labile polymer by its differential sensitivity to nocodazole, and newly stabilized polymer was distinguished from older stable polymer by its differential staining for tyrosinated $\alpha$-tubulin. Our results indicate that the distal axon contains only a slightly higher proportion of newly stabilized polymer than does the axon shaft, but that the proximal axon contains significantly higher levels of newly stabilized polymer than does either the axon shaft or distal region.

\section{Materials and Methods}

Cell culture. Cultures of rat sympathetic neurons were prepared as follows. Superior cervical ganglia were dissected from newborn rat pups, treated with $0.25 \mathrm{mg} / \mathrm{ml}$ collagenase for $1 \mathrm{hr}$ followed by $0.25 \mathrm{mg} / \mathrm{ml}$ trypsin for $45 \mathrm{~min}$, and then triturated with a Pasteur pipette into a single-cell dispersion. The neurons were then plated onto collagen-coated $35 \mathrm{~mm}$ tissue culture dishes in $\mathrm{N} 2$ medium (Moya et al., 1980) supplemented with $2.5 \%$ fetal bovine serum, $5 \%$ human placental serum, $50 \mathrm{ng} / \mathrm{ml}$ nerve growth factor, and $0.6 \%$ methyl cellulose. The neurons were plated at a relatively low density ( $3 / 4$ of a ganglion per dish) to reduce axon fasciculation and limit dendritic outgrowth (Higgins et al., 1991). The next morning, cultures were fed with the same medium without the methyl cellulose, but containing $5 \mu \mathrm{M}$ cytosine arabinoside to reduce non-neuronal contamination. Experiments were performed on the third day in culture, by which time the axons had grown to be several hundred microns in length.

Effects of nocodazole on axonal microtubules. An important goal of the present study was to determine the stability properties of MTs in the proximal and distal regions of the axon and the axon shaft (see introductory remarks). To accomplish this, we quantified the effects of nocodazole, a potent and highly specific MT poison, as described in two previous studies (Baas and Black, 1990; Baas et al., 1991). Briefly, cultures were treated for times ranging between 0 and 360 min with 2 $\mu \mathrm{g} / \mathrm{ml}$ nocodazole (Aldrich Chemical Co., Milwaukee, WI), and were then prepared for standard electron microscopy. After embedment in resin, proximal and distal regions of the axons and regions of the axon shaft were selected by phase-contrast microscopy, sectioned parallel to the substratum, viewed electron microscopically, and photographed. Total lengths of MT profiles were mcasured separately for cach of these three axon regions in the electron micrographs for each time point, and standardized to unit area of axoplasm. For each axon region and experimental condition, a minimum of 10 axons were analyzed. The MT mass in the three regions after drug treatment was then expressed as a function of MT mass in corresponding regions of control axons.

Modeling of the kinetics of drug-induced MT depolymerization. As in our previous study (Baas et al., 1991), we used a modeling approach to test the hypothesis that there are two types of MT polymer in the axon that differ in their kinetics of drug-induced depolymerization. The data for the decline in MT mass with time in drug were fitted to a twocomponent model that is described by a linear combination of two exponential functions of the general form

$$
y=\left(A e^{-B t}\right)+\left(C e^{-D t}\right)
$$

where $y=\% \mathrm{MT}$ mass and $t=$ time in drug. In our analyses, the expression $A e^{-B t}$ describes the kinetics for the more rapidly declining component (phase I) and the expression $\mathrm{Ce}^{-D t}$ describes the kinetics for the more slowly declining component (phase II). The coefficients for phase II ( $C$ and $D$ in Eq. 1$)$ and phase I $(A$ and $B$ ) were obtained by applying an exponential least-squares curve fit to the data (Baas et al., 1991). More information is provided in the Figure 5 caption.

Immunoelectron microscopy. Immunoelectron microscopic visualization of tyrosinated $\alpha$-tubulin in axonal MTs was performed as previously described (Baas and Black, 1990). Briefly, cultures were rinsed in PHEM (60 mm PIPES, $25 \mathrm{~mm}$ HEPES, $10 \mathrm{~mm}$ EGTA, $2 \mathrm{~mm} \mathrm{MgCl}_{2}$, $\mathrm{pH}$ 6.9), extracted for $10 \mathrm{~min}$ with $1 \%$ Triton X-100 in PHEM also containing $10 \mu \mathrm{M}$ taxol and $0.2 \mathrm{M} \mathrm{NaCl}$, and then fixed by the addition of equal volume of PHEM containing $1 \%$ glutaraldehyde (Polysciences Inc., Warrington, PA) directly to the extraction buffer. After 10 min of fixation, cultures were treated with $2 \mathrm{mg} / \mathrm{ml}$ sodium borohydride in PHEM for $15 \mathrm{~min}$, incubated in a blocking solution, and then with primary antibody overnight at $4^{\circ} \mathrm{C}$. The following morning, cultures were rinsed extensively, incubated in gold-conjugated second antibody for $3 \mathrm{hr}$ at $37^{\circ} \mathrm{C}$, rinsed extensively again, and then prepared for electron microscopy by conventional means. The primary antibody used in these analyses was $Y L 1 / 2$, a rat monoclonal against tyrosinated $\alpha$-tubulin (Kilmartin et al., 1982; Wheland et al., 1983), purchased from Accurate Chemical and Scientific Corporation (Westbury, NY) and used at a concentration of 1:200. The second antibody was a $5 \mathrm{~nm}$ gold-conjugated goat anti-rat antibody manufactured by Jansen, purchased from Amersham (Arlington Heights, IL), and used at a concentration of 1:2.

Selection and relocation of axon regions for electron microscopy. Our analyses of the three regions of the axon were contingent upon our ability to relocate specifically the proximal and distal regions of the axon and regions of the axon shaft with the electron microscope. The standard technique in our laboratory for accomplishing such relocations is as follows. First, the area of interest is selected using phase optics on the Epon-embedded sample. This region is then placed in the center of the viewing field, and is circled on the Epon using a diamond-marker objective. The smallest possible circle is made to limit the inclusion of other biological material, and either a sketch or a photograph of the circled region is prepared. A blockface is then carved from the circled region and sectioned, and the sections are viewed with the electron microscope. The sketch or photograph is used to assist in the relocation of the precise axon region of interest.

\section{Results}

Drug-stable and drug-labile MT polymer are present in all three regions of the axon. Sensitivity to nocodazole, a potent MTdepolymerizing drug, was used to evaluate the stability properties of MTs in three regions of the axon: the $10 \mu \mathrm{m}$ closest to the growth cone (distal axon), the $10 \mu \mathrm{m}$ closest to the cell body (proximal axon), and the axon shaft, more than $100 \mu \mathrm{m}$ from both the cell body and the growth cone (see Fig. 1). After treatment with nocodazole for varying times, the MT mass remaining in each of the three regions was quantified morphometrically and expressed as a percentage of the MT mass in control cultures. Electron micrographs of control and drug-treated axons are shown in Figure 2. A progressive loss of MT polymer was observed from all three regions of the axon as a function of time in drug. Quantitative analyses of this decline are shown in Figure 3 (top row), in which the data are displayed on semi-log plots. In all three axon regions, the MT mass declined biphasically, suggesting that the total polymer in each region consists of two distinct components that differ in their depolymerization kinetics. We have previously established that treatment with nocodazole results in a biphasic decline in the MT mass in the axon shaft, and that this decline can be modeled as a linear combination of two exponential functions (Baas et al., 1991). This same type of model also effectively describes the nocodazole-induced decrease in MT polymer for the three axon regions examined in the present studies (see Fig. 3). Thus, we conclude 


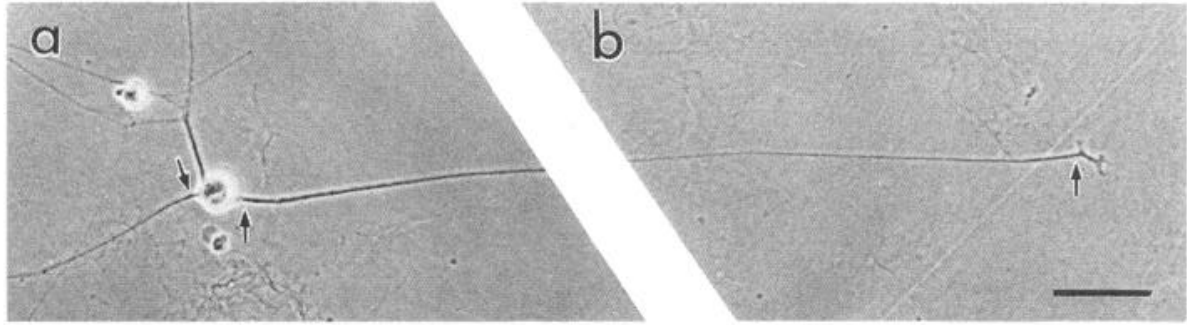

Figure 1. Phase-contrast micrographs of a rat sympathetic neuron cultured as described in Materials and Methods. $a$ and $b$ show the proximal and distal regions of different axons, all of which were several hundred microns long. $A r$ rows in $a$ indicate the proximal regions of axons, while arrow in $b$ indicates the distal region of the axon. Scale bar, 50 $\mu \mathrm{m}$. that the proximal and distal regions of the axon, like the shaft, contain two types of MT polymer that differ in their rates of drug-induced depolymerization.

Using the equation for the two-component model, we extracted the "pure" kinetics for the individual components in each of the three regions of the axon (see Materials and Methods). The broken lines in the bottom row of Figure 3 show the resulting theoretical curves for the more rapidly depolymerizing polymer (phase I), and the more slowly depolymerizing polymer (phase II) for all three regions of the axon. In the proximal axon, phase I and phase II account for $55 \%$ and $45 \%$ of the MT mass, respectively, and these phases decline with half-times of $5.1 \mathrm{~min}$ and $139 \mathrm{~min}$, respectively. In the distal axon, phase I and phase II account for $95 \%$ and $5 \%$ of the MT mass, respectively, and these phases decline with half-times of $2.9 \mathrm{~min}$ and $107 \mathrm{~min}$, respectively. In the axon shaft, phase I and phase II account for

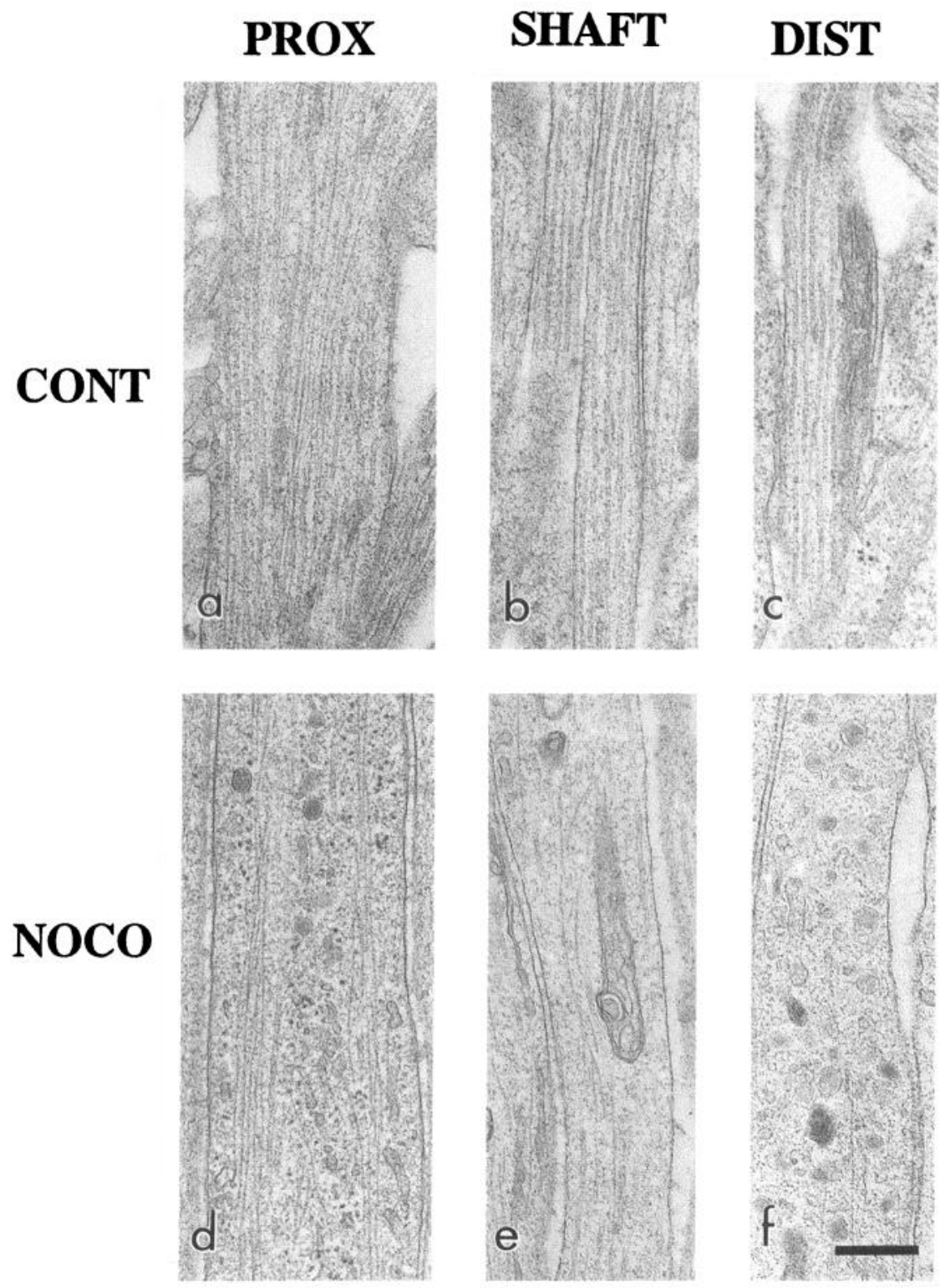

Figure 2. Transmission electron micrographs of the proximal, distal, and shaft regions of different axons. $a-c$ are from control cultures, while $d-f$ are from cultures that had been treated with 2 $\mu \mathrm{g} / \mathrm{ml}$ nocodazole for $30 \mathrm{~min}$. A decrease in MT mass is apparent in all three regions of the drug-treated axons. Scale bar, $0.3 \mu \mathrm{m}$. 

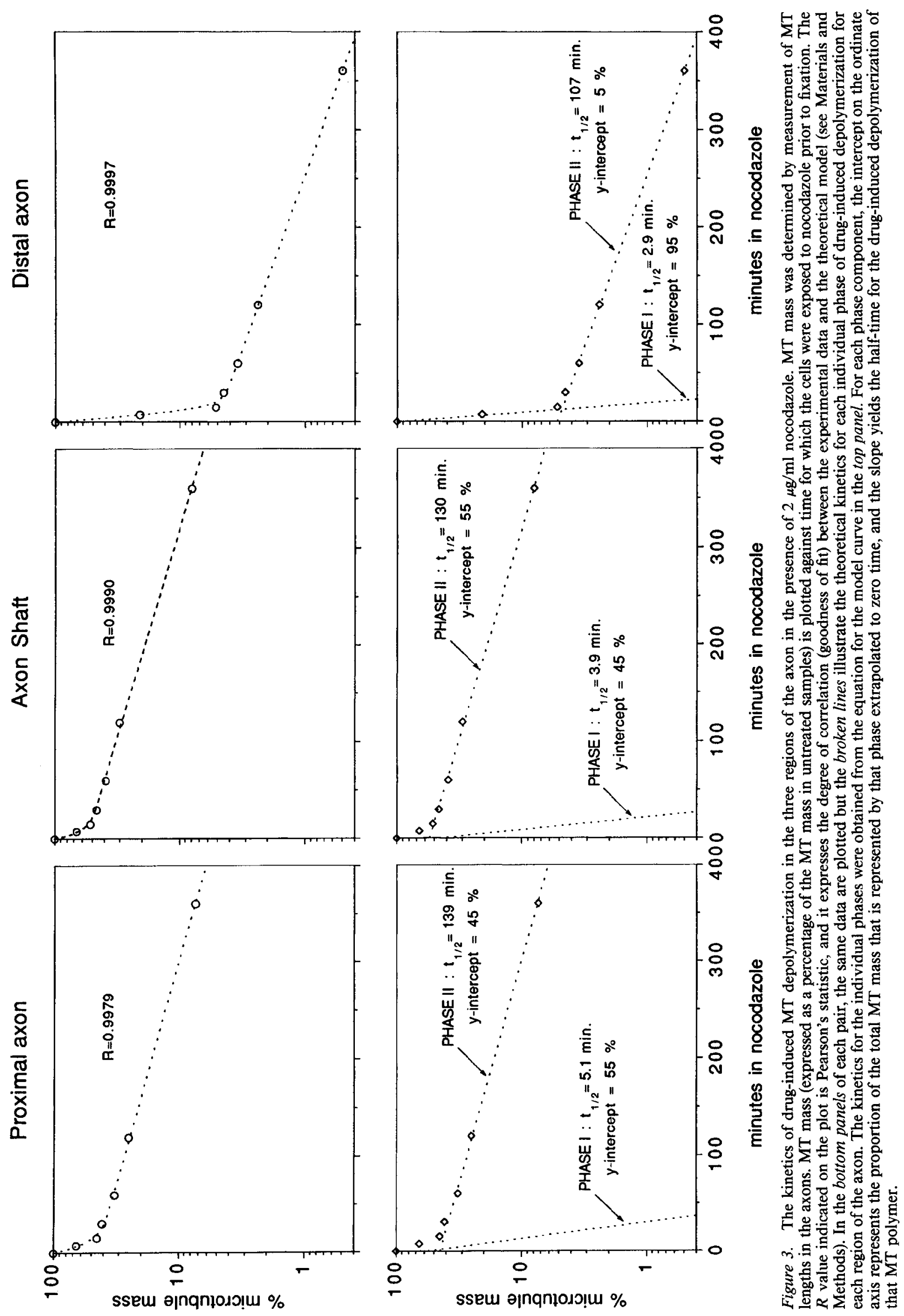
Table 1.

A. Density of total, labile, and stable MT polymer

( $\mu \mathrm{m} \mathrm{MT} / \mu \mathrm{m}^{2}$ axoplasm)

\begin{tabular}{llll} 
& Proximal axon Axon shaft & Distal axon \\
\hline Total polymer & $4.74(100 \%)^{a}$ & $3.96(100 \%)$ & $4.15(100 \%)$ \\
Labile polymer & $2.65(55 \%)$ & $1.79(45 \%)$ & $3.94(95 \%)$ \\
Stable polymer & $2.12(45 \%)$ & $2.18(55 \%)$ & $0.22(5 \%)$ \\
\hline
\end{tabular}

${ }^{a}$ Percentage of total polymer.

B. Percentage of labile and stable MT polymer remaining after 30 min in nocodazole

\begin{tabular}{|c|c|c|c|}
\hline & Proximal axon & Axon shaft & Distal axon \\
\hline Labile polymer ${ }^{b}$ & $1.71 \%$ & $0.50 \%$ & $0.07 \%$ \\
\hline Stable polymer & $86.1 \%$ & $85.2 \%$ & $82.3 \%$ \\
\hline
\end{tabular}

${ }^{b}$ Percentage of labile polymer.

'Percentage of stable polymer.

Quantitative data on (in part $A$ ) the density of total, drug-labile, and drug-stable MT polymer in the proximal, shaft, and distal regions of the axon, and (in part $B$ ) the percentage of drug-labile and drug-stable polymer remaining in each of these regions after $30 \mathrm{~min}$ in $2 \mu \mathrm{g} / \mathrm{ml}$ nocodazole. Raw data were derived from summing the total lengths of MT polymer per square micron of axoplasm in clcctron micrographs of cach of the three regions of control and nocodazole treated axons. The data were then analyzed as previously described (Baas et al, 1991; see Results). A minimum of $40 \mu \mathrm{m}^{2}$ of axoplasm was analyzed for each experimental condition.

$45 \%$ and $55 \%$ for the MT mass, respectively, and these phases decline with half-times of $3.9 \mathrm{~min}$ and $130 \mathrm{~min}$, respectively. In the present study, as in our previous work, we refer to the more rapidly depolymerizing polymer (phase I) as drug labile, and the more slowly depolymerizing polymer (phase II) as drug stable.

These studies reveal both striking similarities as well as differences in the stability properties of the MT polymer in the three axon regions. All three regions contain drug-labile and drug-stable polymer. Furthermore, the drug-labile MT polymer is very similar in all three regions with regard to its depolymerization kinetics, and the same is true for the drug-stable polymer. We cannot evaluate the significance of the small differences in the depolymerization kinetics among the axon regions. The principal difference concerns the proportions of drug-labile and drug-stable polymer, which are very different in the distal axon compared to both the proximal axon and axon shaft, and are slightly different in the proximal axon compared to the shaft (for summary, see Table 1).

Quantification of newly stabilized MT polymer in the axon. The principal goal of the present study was to identify the site(s) of MT stabilization for the axon. To accomplish this, we determined the amounts of newly stabilized MT polymer in different regions of the axon, reasoning that those regions that are particularly rich in newly stabilized polymer will correspond to major sites of MT stabilization for the axon. We took advantage of the relationship between the stability properties of MTs and their tyrosination state to identify newly stabilized polymer. We previously showed that drug-labile polymer stains densely for tyrosinated $\alpha$-tubulin, whereas the majority of the drug-stable polymer docs not stain. Bccause stable polymer is generated from labile polymer, the most recently stabilized polymer should still contain relatively high levels of tyrosinated $\alpha$-tubulin. Accordingly, we have defined the newly stabilized MT polymer as
Table 2. Amount of MT polymer labeled for tyrosinated tubulin after 0 and $30 \mathrm{~min}$ in nocodazole

$\mu \mathrm{m} \mathrm{MT} / \mu \mathrm{m}^{2}$ axoplasm

( $\%$ of initial MT polymer)

\begin{tabular}{|c|c|c|c|}
\hline & Proximal axon & Axon shaft & Distal axon \\
\hline \multicolumn{4}{|l|}{0 min nocodazole } \\
\hline Total polymer & $4.74(100 \%)$ & $3.96(100 \%)$ & $4.15(100 \%)$ \\
\hline Labeled polymer & $4.36(92 \%)$ & $1.96(50 \%)$ & $4.15(100 \%)$ \\
\hline Unlabeled polymer & $0.38(8 \%)$ & $2.00(50 \%)$ & $0.00(0 \%)$ \\
\hline \multicolumn{4}{|l|}{30 min nocodazole } \\
\hline Total polymer & $1.95(41 \%)$ & $1.83(46 \%)$ & $0.19(4.5 \%)$ \\
\hline Labeled polymer & $1.53(32 \%)$ & $0.09(2.3 \%)$ & $0.19(4.5 \%)$ \\
\hline Unlabeled polymer & $0.42(8.8 \%)$ & $1.74(44 \%)$ & $0.00(0 \%)$ \\
\hline
\end{tabular}

Data are from calculations on the amount of MT polymer that labels for tyrosinated $\alpha$-tubulin in the proximal, shaft, and distal axon regions in immunoelectron microscopic analyses of cultures treated with $2 \mu \mathrm{g} / \mathrm{ml}$ nocodazole for 0 and 30 min. Calculations were derived from information on the levels of drug-stable and drug-labile polymer in each axon region (Table 1), considered in combination with information on the levels of tyrosinated polymer before and after drug treatment. See Results for more details.

drug-stable polymer that stains densely for tyrosinated $\alpha$-tubulin.

Immunoelectron microscopic analyses of MTs in control axons reveal that $50 \%$ of the polymer in the axon shaft stains for tyrosinated $\alpha$-tubulin, whereas $92 \%$ and $100 \%$ of the polymer stains in the proximal and distal regions, respectively (Fig. 4, Table 2). In all three regions, this proportion is greater than the proportion of polymer that is drug labile (compare Tables 1, 2). Thus, some of the polymer that stains for tyrosinated $\alpha$-tubulin in the proximal and distal regions of the axon and the axon shaft is drug stable. Assuming that all of the drug-labile polymer labels for tyrosinated $\alpha$-tubulin, we can calculate the proportion of total polymer in the three axon regions that is both drug stable and tyrosinated based on known proportions of druglabile and drug-stable polymer in these regions (Table 1) and on the proportions of total polymer in these regions that label for tyrosinated $\alpha$-tubulin (Table 2) (i.e., the levels of polymer that is both drug stable and tyrosinated can be calculated based on these data alone, without having to perform immunoelectron microscopy on drug-treated cultures). These calculations indicate that $36 \%, 4 \%$, and $5 \%$ of the total polymer in the proximal, shaft, and distal regions of the axon, respectively, is drug stable and tyrosinated, and thus, by our definition, is newly stabilized.

We have directly measured the amount of drug-stable polymer that is tyrosinated by performing immunoelectron microscopic analyses on neurons in which the drug-labile polymer was dissected away from the drug-stable polymer by treatment with nocodazole for $30 \mathrm{~min}$. This treatment reduced the amount of drug-labile polymer in each axon region by $>98 \%$, but left $>82 \%$ of the drug-stable polymer remaining (Table 1). Thus, the resulting preparation contains almost exclusively drug-stable MT polymer and at amounts only modestly reduced compared to untreated neurons. Immunoelectron microscopic staining of these preparations for tyrosinated $\alpha$-tubulin revealed that a portion of the remaining polymer in each axon region labeled densely for tyrosinated $\alpha$-tubulin (Fig. 4, Table 2). The amount of this labeled polymer greatly exceeded the low levels of druglabile polymer remaining in thesc drug-trcated axons (by factors of 34,10 , and 68 for the proximal, shaft, and distal regions of the axon, respectively). Thus, all regions of the axon contain polymer that is both drug stable and tyrosinated. 
PROX

\section{CONT}

Figure 4. Electron micrographs of MTs from the proximal, distal, and main shaft regions of different axons immunostained with the YL 1/2 antibody against tyrosinated $\alpha$-tubulin and an appropriate second antibody conjugated to $5 \mathrm{~nm}$ colloidal gold. $a-c$ are from control cultures, while $d-f$ are from cultures that had been treated for 30 min with $2 \mu \mathrm{g} / \mathrm{ml}$ nocodazole. In control axons, the shaft contains roughly equal levels of labeled and unlabeled polymer, while both the proximal and distal regions contain predominantly labeled polymer. After drug treatment, a decrease in MT mass is apparent in all three regions of the axon. After drug treatment, the polymer remaining in the axon shaft is predominantly unlabeled, while the polymer remaining in the proximal axon is predominantly labeled. Little or no polymer remains in the distal axon after drug treatment. Quantitative data from these studies are represented in Figure 5. Scale bar, 0.3 $\mu \mathrm{m}$.
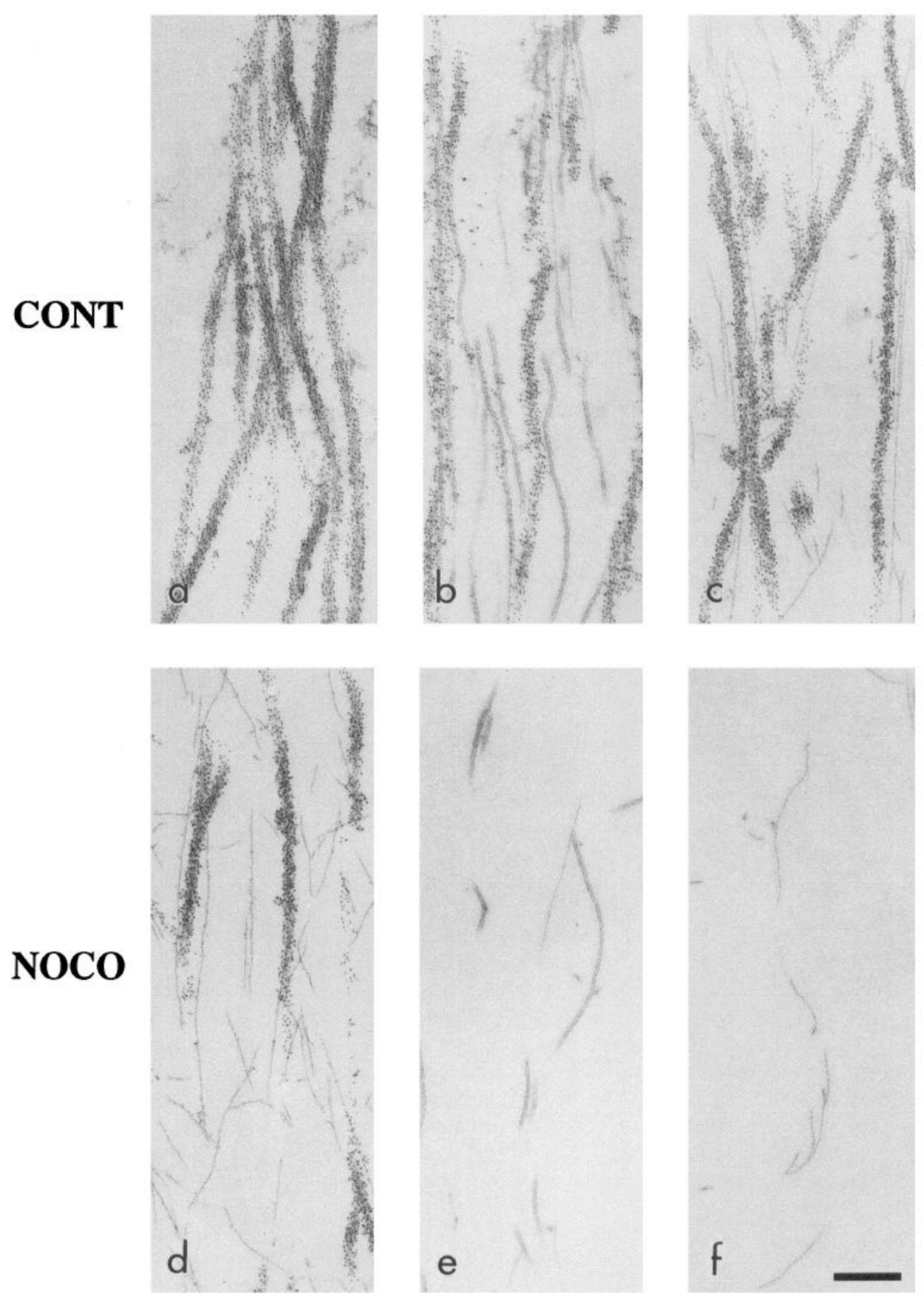

The amounts of labeled polymer in the three regions of drugtreated axons (Table 2) are slightly less than the values calculated on the basis of MT staining for tyrosinated $\alpha$-tubulin in control axons (see above). This probably reflects the fact that a portion of the drug-stable polymer lost during the drug treatment (Table 1) was tyrosinated. To evaluate this possibility, we have corrected the amount of labeled polymer in drug-treated axons for the amount of drug-stable polymer lost during drug treatment, and also for the contribution of the limited amount of drug- labile MT polymer that remains after drug treatment (see Fig. 5 caption). After correction, $36.4 \%, 2.4 \%$, and $5.4 \%$ of total polymer in the proximal, shaft, and distal regions of the axon, respectively, is both tyrosinated and drug stable. These values agree very well with those determined above. It is noteworthy that the proportion of tyrosinated drug-stable polymer varies within the axon. The proportion is greatest in the proximal axon, exceeding the amounts in the axon shaft and distal axon by factors of $\approx 15$-fold and $\approx 7$-fold, respectively. 


\section{Discussion}

In the present study, we sought to determine the principal site(s) where axonal MTs are stabilized by quantifying the proportion of polymer that is newly stabilized in three different regions of the axon. Newly stabilized polymer was defined as that portion of the polymer that depolymerizes more slowly in the presence of nocodazole and that stains densely for tyrosinated $\alpha$-tubulin in immunoelectron microscopic analyses (see introductory remarks and Results). The reliability of these criteria to distinguish newly stabilized polymer is contingent upon certain assumptions. The first is that nocodazole sensitivity accurately reflects the physiological stability and hence the age of the MT polymer. While there is cxpcrimental cvidence that supports this vicw (see, e.g., Kreis, 1987), it must be acknowledged that many features of the drug's pharmacology are still unknown. The second is that tubulin carboxypeptidase, the enzyme responsible for detyrosinating $\alpha$-tubulin, is uniformly active throughout the axon. Support for this assumption derives from observations that MT polymer throughout the neuron stains with an antibody specific for detyrosinated $\alpha$-tubulin in both immunofluorescence and immunoelectron microscopic assays (Gunderson and $\mathrm{Bu}-$ linski, 1986; Lim et al., 1989; Baas et al., 1991), and that treatment with taxol leads to a progressive decrease in the tyrosinated $\alpha$-tubulin content of MTs throughout the axon (Robson and Burgoyne, 1989; Arregui et al., 1991; Brown et al., 1992). Thus, available information supports the legitimacy of our criteria for identifying newly stabilized polymer.

Using these criteria, we observed clear differences with regard to the content of newly stabilized MT polymer in the proximal and distal regions of the axon and the axon shaft. Newly stabilized polymer is present throughout the axon. However, a severalfold greater proportion of the total polymer in the most proximal $10 \mu \mathrm{m}$ of the axon is newly stabilized compared to an equivalent length of the axon shaft or distal region of the axon. Thus, it appears that MT stabilization occurs all along the length of the axon, but that the proximal region is by far the most active with regard to this process.

The present findings alone do not reveal the extent to which the proximal axon contributes to the levels of newly stabilized polymer within the entire axon. To explore this issue further, we have performed calculations also taking into account the quantitative immunofluorescence analyses of Brown et al. (1992) on the levels of total and tyrosinated MT polymer along the length of the entire axon. First, we used the immunofluorescence data to obtain a relative measure of the amount of total MT polymer as a function of position along the lengths of axons averaging $350 \mu \mathrm{m}$ in length. A relative measure of the total polymer in each of the regions was determined from intensity measurements of $\beta$-tubulin immunofluorescence. Then, the amount of newly stabilized polymer in each region was calculated using the present data on the proportion of newly stabilized polymer in the proximal, shaft, and distal regions of the axon. Our calculations indicate that for the 53 axons analyzed in the previous study, $52 \pm 13 \%$ of the total newly stabilized polymer is present in the most proximal $10 \mu \mathrm{m}, 46 \pm 12 \%$ is present along the length of the entire axon shaft, and only $2+3 \%$ is present in the most distal $10 \mu \mathrm{m}$. Thus, for axons that are roughly $350 \mu \mathrm{m}$ long, approximately half of the total amount of newly stabilized MT polymer is present in the most proximal $10 \mu \mathrm{m}$, a region that represents less than $3 \%$ of the total length of the axon. The contribution of the proximal axon to the total newly
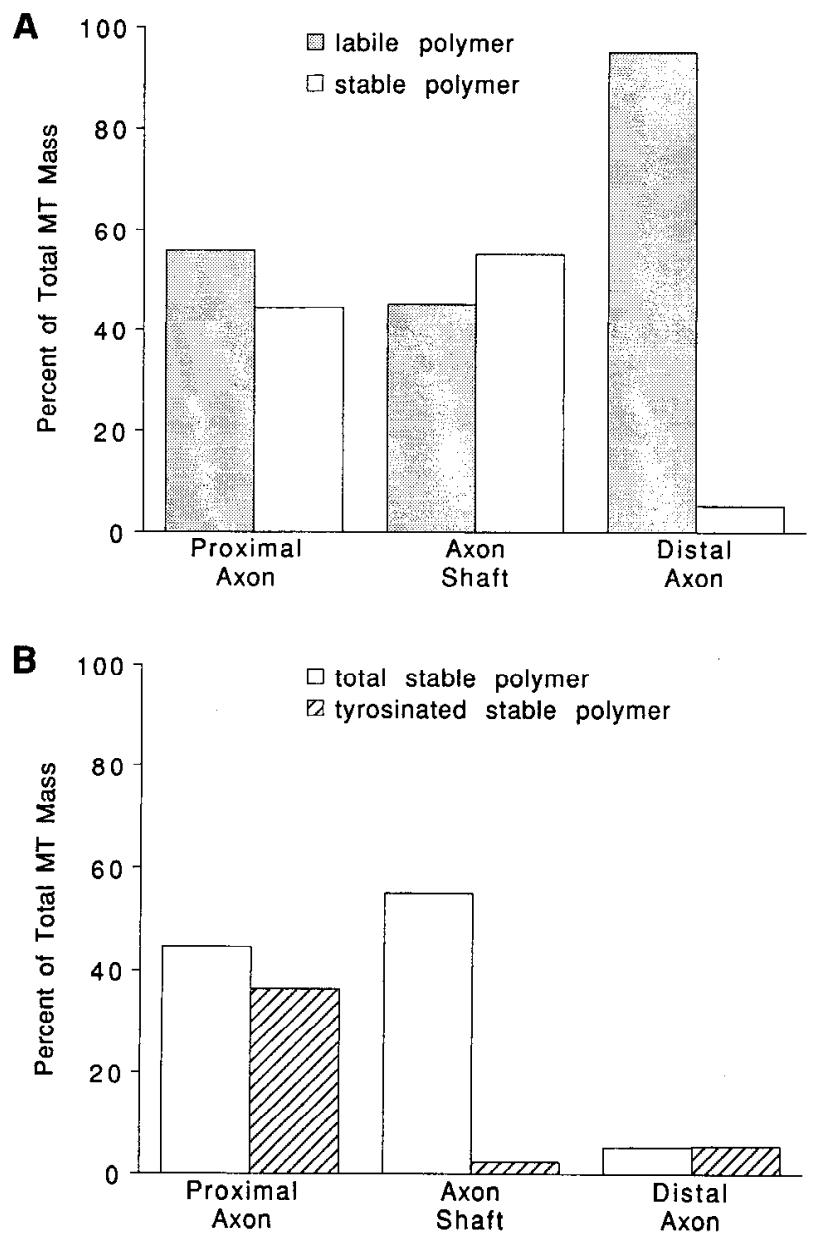

Figure 5. A shows the percentage of total polymer in each axon region that is drug labile (shaded bars) or drug stable (open bars). The data are taken from Table 1. $B$ shows the proportion of total polymer that is drug stable (open bars) or that is both drug stable and tyrosinated (hatched bars). These latter values were calculated by correcting the amount of tyrosinated polymer in axons treated with nocodazole for $30 \mathrm{~min}$ (see Table 2) for the contribution of drug-labile polymer and also for the amount of tyrosinated drug-stable polymer lost during drug treatment. To correct for the contribution of drug-labile polymer, we have assumed that all of the drug-labile polymer remaining in the drug-treated axons stains for tyrosinated $\alpha$-tubulin, and accordingly have subtracted the amount of drug-labile polymer remaining (see Table 1) from the amount of total labeled polymer in the drug-treated axons. To correct for the amount of tyrosinated drug-stable polymer lost during drug treatment, we have assumed that tyrosinated drug-stable polymer and detyrosinated drug-stable polymer have identical susceptibility to nocodazole treatment. Our analyses of the kinetics of polymer loss in the presence of drug (see Fig. 3) support this assumption because the decline in drugstable polymer during drug treatment best fits a single-component model. This result would not have been obtained if tyrosinated drug-stable polymer and detyrosinated drug-stable polymer were appreciably different in their kinetics of decline in the presence of drug. Accordingly, after correcting the amount of labeled polymer in drug-treated axons for the contribution of drug-labile polymer, we calculated the proportion of drug-stable polymer remaining in each region that stained for tyrosinated $\alpha$-tubulin. The resulting value was then used to calculate the amount of tyrosinated drug-stable polymer lost during drug treatment, based on the amount of total drug-stable polymer lost (see Table 1). This latter value was added to the amount of tyrosinated drug-stable polymer remaining after drug treatment to obtain the corrected value for the total amount of tyrosinated drug-stable polymer. 
stabilized polymer may be even higher than $50 \%$ if the actual length of the proximal axon that contains elevated levels of newly stabilized polymer actually extends beyond the first 10 $\mu \mathrm{m}$. Consistent with this notion, the quantitative immunofluorescence studies have defined a region of the proximal axon roughly $40 \mu \mathrm{m}$ in length in which the tyrosinated $\alpha$-tubulin content of the polymer exceeds that in the axon shaft (Brown et al., 1992). If this entire region contains levels of newly stabilized polymer comparable to those in the most proximal 10 $\mu \mathrm{m}$, then this extended region, which represents $11 \%$ of the length of a $350 \mu \mathrm{m}$ axon, contains $\approx 75 \%$ of the newly stabilized polymer in the entire axon.

It is interesting to note that, despite the relatively low MT stabilizing activity within the axon shaft, drug-stable polymer comprises $\approx 50 \%$ of the total polymer within that region of the axon, and this appears to be correct even for very young axons. A possible explanation for this discordance is that much of the drug-stable polymer in the axon shaft was not stabilized within the shaft itself, but arrived there after its stabilization elsewhere. In this regard, the proximal axon, with its very high stabilizing activity, is a likely candidate for the source of much of this drugstable polymer in the axon shaft. If much of the drug-stable polymer in the shaft originates within the proximal axon, then the proximal axon is a principal site for the net addition of drugstable polymer for the entire axon. Moreover, if all of this reasoning is correct, then it is necessary to conclude that mechanisms exist within the axon to transport the drug-stable polymer generated proximally to more distal sites throughout the axon. Therefore, although in a rather indirect manner, our results support the long-standing polymer translocation model for axon growth (Lasek, 1982, 1986, 1988). Direct support for this model, which was originally based on the transport kinetics of tubulin in the axon, has derived from studies on the movement of photoactivated bands of tubulin in the axon (Reinsch et al., 1991), and additional indirect support has derived from observations suggesting that the cell body is the source of new MTs for growing axons (Black et al., 1986; Baas and Ahmad, 1992; Baas and Joshi, 1992).

In conclusion, we have investigated the sites where drugstable MT polymer is added to growing axons. Our data indicate that the proximal region of the axon is particularly rich in newly stabilized polymer and hence is a major site for this addition. By contrast, our data show that very little newly stabilized polymer is present within the distal region of growing axons, and hence that very little stable polymer is added distally. In considering these results, it is important to keep in mind that the levels of drug-labile polymer also increase as the axon grows, and the major sites for this addition are still unknown. Clearly, additional efforts will be required to elucidate the precise contributions of each region of the axon to the expansion of its MT array.

\section{References}

Ahmad FJ, Pienkowski TP, Baas PW (1993) Regional differences in microtubule dynamics in the axon. J Neurosci 13:856-866.

Arregui C, Busciglio J, Caceres A, Barra HS (1991) Tyrosinated and detyrosinated microtubules in axonal processes of cerebellar macroneurons grown in culture. J Neurosci Res 28:171-181.

Baas PW, Ahmad FJ (1992) The plus ends of stable microtubules are the exclusive nucleating structures for microtubules in the axon. $J$ Cell Biol 116:1231-1241.

Baas PW, Black MM (1990) Individual microtubules in the axon consist of domains that differ in both composition and stability. $J$ Cell Biol 111:495-509.
Baas PW, Joshi HC (1992) Gamma-tubulin distribution in the neuron: implications for the origins of neuritic microtubules. J Cell Biol 119: 171-178.

Baas PW, Slaughter T, Brown A, Black MM (1991) Microtubule dynamics in axons and dendrites. J Neurosci Res 30:134-153.

Bamburg JR, Bray D, Chapman K (1986) Assembly of microtubules at the tip of growing axons. Nature 321:788-790.

Barra HS, Arce CA, Argarana CE (1988) Post-translational tyrosination/detyrosination of tubulin. Mol Neurobiol 2:133-153.

Black MM, Greene LA (1982) Changes in colchicine susceptibility of microtubules associated with neurite outgrowth: studies with nerve growth factor responsive PC12 pheochromocytoma cells. J Cell Biol 95:379-386.

Black MM, Cochran JM, Kurdyla JT (1984) Solubility properties of neuronal tubulin: evidence for labile and stable microtubules. Brain Res 295:255-265.

Black MM, Keyser P, Sobel E (1986) Interval between the synthesis and assembly of cytoskeletal proteins in cultured neurons. J Neurosci 6:1004-1012

Brady ST, Tytell M, Lasek RJ (1984) Axonal transport and axonal tubulin: biochemical evidence for cold stability. J Cell Biol 99:17161724.

Brown A, Slaughter T, Black MM (1992) Newly assembled microtubules are concentrated in the proximal and distal regions of growing axons. J Cell Biol 119:867-882.

Bulinski JC, Gunderson GG (1991) Stabilization and post-translational modification of microtubules during cellular morphogenesis. Bioessays 13:285-293.

Ferreira A, Busciglio J, Caceres A (1989) Microtubule formation and neurite growth in cerebellar macroneurons which develop in vitro: evidence for the involvement of the microtubule-associated proteins, MAP 1a, HMW-MAP2, and tau. Dev Brain Res 49:215-228.

Gunderson GG, Bulinski JC (1986) Microtubule arrays in differentiated cells contain elevated levels of a post-translationally modified form of tubulin. Eur J Cell Biol 42:288-294.

Gunderson GG, Khawaja S, Bulinski JC (1987) Postpolymerization detyrosination of microtubules: a mechanism for the subcellular differentiation of microtubules. J Cell Biol 105:251-264.

Higgins D, Lein PJ, Osterhout DJ, Johnson MI (1991) Tissue culture of mammalian autonomic neurons. In: Culturing nerve cells (Banker G, Goslin K, eds), pp 177-205. Cambridge, MA: MIT Press.

Khawaja S, Gunderson GG, Bulinski JC (1988) Enhanced stability of microtubules enriched in detyrosinated tubulin is not a direct function of detyrosination level. J Cell Biol 106:141-150.

Kilmartin JV, Wright B, Milstein C (1982) Rat monoclonal antitubulin antibodies derived by using a new nonsecreting rat cell line. J Cell Biol 93:576-582.

Kirschner M, Mitchison T (1986) Beyond self-assembly: from microtubules to morphogenesis. Cell 45:329-342.

Kreis TE (1987) Microtubules containing detyrosinated tubulin are less dynamic. EMBO J 6:576-582.

Lasek RJ (1982) Translocation of the neuronal cytoskeleton and axonal locomotion. Philos Trans R Soc Lond [Biol] 299:313-327.

Lasek RJ (1986) Polymer sliding in axons. J Cell Sci [Suppl] 5:161179.

Lasek RJ (1988) Studying the intrinsic determinants of neuronal form and function. In: Intrinsic determinants of neuronal form and function (Lasek RJ, Black MM, eds), pp 1-58. New York: Liss.

Lim S-S, Sammak PJ, Borisy GG (1989) Progressive and spatially differentiated stability of microtubules in developing neuronal cells. J Cell Biol 109:253-264.

Mitchison T, Kirschner M (1988) Cytosketelal dynamics and nerve growth. Neuron 1:761-772.

Morris JR, Lasek RJ (1984) Monomer-polymer equilibria in the axon: direct measurement of tubulin and actin as polymer and monomer in axoplasm. J Cell Biol 107:2064-2076.

Moya F, Bunge MB, Bunge RP (1980) Schwann cells proliferate but fail to differentiate in defined medium. Proc Natl Acad Sci USA 77: 6902-6906.

Reinsch SS, Mitchison TJ, Kirschner MW (1991) Microtubule polymer assembly and transport during axonal elongation. J Cell Biol 115: 365-380.

Robson SJ, Burgoyne RD (1988) Differential levels of tyrosinated, detyrosinated, and acetylated alpha-tubulins in neurites and growth cones of dorsal-root ganglion neurons. Cell Motil Cytoskel 8:155164. 
Webster DR, Wheland J, Weber K, Borisy GG (1990) Detyrosination of alpha tubulin does not stabilize microtubules in vivo. J Cell Biol 111:113-122.

Wheland J, Willingham MC, Sandoval IV (1983) A rat monoclonal antibody reacting specifically with the tyrosinated form of $\alpha$-tubulin. Biochemical characterization. Effects on microtubule polymerization in vitro and microtubule polymerization and organization in vivo. $\mathrm{J}$ Cell Biol 97:1476-1490. 